\title{
Research on Fault-Tolerant Routing Mechanism of NoC
}

\author{
Guowei Hou ${ }^{1, \text { a }}$, Lixin $\mathrm{Yu}^{2, \mathrm{~b}}$, Liguo Song ${ }^{3, \mathrm{c}}$, Heping Peng ${ }^{3, \mathrm{c}}$, Wei Zhuang ${ }^{3, \mathrm{c}}$ \\ ${ }^{1,2,3}$ North road No.2, Siyingmen, Donggaodi, Fengtai district, Beijing, China \\ amaxhou@163.com, b745845791@qq.com, '15911106595@163.com
}

Keywords: Network-on-Chip (NoC); reliability; fault-tolerant; hybrid error detection; retransmission

\begin{abstract}
With reducing feature size of transistors and increasing of cores on a single chip, to overcome poor scalability and bandwidth limitation on a single bus, Network-on-Chip (NoC) has been proposed. However, reliability of Multiprocessor system based on Network-on-Chip (NoC) has become a significant challenge due to the transmission fault. The paper analyzes some fault-tolerant Routing methods based on retransmission, and hybrid error detection and retransmission fault-tolerant scheme is presented. Instead of using a simplex end-to-end (e2e) or router-to-router (r2r) error detection, the paper utilizes both of these two mechanisms which means head flit uses r2r while data flit uses e2e retransmission. To make the cost lower, in r2r error detection and retransmission operation, the paper employs comparator instead of encoder/decoder. At last, the advantages and disadvantages of different retransmission fault-tolerant routing mechanism are analyzed and the trend of this field is proposed.
\end{abstract}

\section{Introduction}

With the reduction in feature size of semiconductor and according to Moore's Law, System on Chip (SoC) designs are enable to be integrated complicated with large number of cores and resources. To gain the maximum utilization of these resources, they need to be connected through an environment that enables rapid inter-exchange of a large number of data. Traditional bus and crossbar architecture can no longer satisfy the increasing scalability needs and the growing bandwidth requirement in Multiprocessor System on Chip (MPSoC) [1]. Then Network on Chip (NoC) is proposed. Since now, NoC has been employed in many fields such as electronic industry, satellite communications and spaceflight. However, there are several challenges faced by NoC like performance, power and reliability. Thereinto, reliability is becoming a critical issue as we enter the deep submicron area, especially in spaceflight application field.

Due to the complex space environment, the reliability of NoC will be affected seriously. Errors caused by cosmic radiation, crosstalk, electromigration, and Single Event Upset (SEU) are common. In addition, several factors including high operation frequency, low voltage, small noise margins and reduced logic depth also contribute to such errors [2].

Traditionally, error detection and correction mechanisms are used to protect communication subsystems against the effects of transient faults which include end-to-end (e2e) retransmission and router-to-router (r2r) retransmission. Other approaches for fault-tolerant methods consist of stochastic communication, adaptive routing [3].

In this paper, a mended fault-tolerant mechanism based on hybrid error detection and retransmission is presented. Instead of using single e2e or r2r error detection scheme, this paper employs hybrid retransmission as representations. As head flit contains some significant message, so it uses r2r error detection to guarantee the data integrity while data flits employ e2e error detection. Importantly, comparator cell is imposed instead of encoder/decoder in each router node in traditional r2r operation to get a better tradeoff between reliability and cost.

The rest of the paper is organized as follows. Section 2 provides an overview of some fault-tolerant methods for NoC. Section 3 shows two typical retransmission schemes and analyzes their advantages and disadvantages separately. Section 4 presents the new hybrid error detection and retransmission 
mechanism. Section 5 shows the practical application of the fault-tolerant scheme. Section 6 offers conclusion and future work.

\section{Research Actuality}

With the increasing integration and importance of MPSoC, the reliability and fault-tolerance of NoC is becoming a new hotspot of research. Many conferences and magazines have published some research results related to fault-tolerance of NoC.

In 2003, Heiko Zimmer [4] presents that the errors of a large number of parallel lines between adjacent routers are instantaneous, and will not occur in the same line. Then he proposes a fault model notation. Zimmer and his partners divide up the data packet and code them respectively, and they also emphasize the significance of head flit to guarantee the Quality-of-Service (QoS).

Bertozzi [5] in Bologna presents a fault-tolerance scheme based on Xpipes architecture. Transfer will send data to receiver. After destination node receives the right flits, it will send a feedback signal to the source which indicates that the transmission is over without errors. If the right data is not receive exactly, the receiver will also send a feedback which manifest that this transmission is incorrect and waive the received data after that until receive the right data.

Murali [6] explores some traditional error recovery methods which include end-to-end error detection, router-to-router error detection and random communication scheme.

Haibo Zhu [7] and his partners pay their attentions to find solutions for reducing infection of data transfer by crosstalk and decreasing the energy cost in transmission line. They presented a crosstalk fault-tolerant mechanism that is suitable to NoC.

\section{Typical Error Detection and Retransmission Fault-Tolerant Routing Mechanism}

As can be seen, NoC not only cares about how the data is transferred from the source to the destination, but also considers the reliability of data transfer. Errors that occur in a NoC based system can be divided into permanent errors and transient errors. To solve the transient errors in the transmission line, fault-tolerant schemes are introduced. In many systems today, Error Checking Code (ECC) is used for error detection, and recovery schemes are invoked to correct these errors. There are two typical retransmission techniques to recover from errors: end-to-end retransmission and router-to-router retransmission [8]

End-to-End Error Detection and Retransmission. In the e2e mechanism [9], during one data packet transfer, error detection will be executed once just at the destination node. Data that needs to be transferred will be encoded by ECC encoders before being transferred into the network at source node. After encoded, the data will be saved temperately and sent to the network to be transferred. At the destination, the data will be decoded and detected. If some errors are found, the destination will send a feedback signal that stands for a false transmission to the source node, and the data will be retransmitted. In this scheme, a correct copy of the data packet will be retransmitted until the packet is received by the destination without any errors. At last, the destination node will send a feedback signal that indicates a correct transmission to the source node to inform that the next packet can begin to be transferred. Fig. 1 shows the architecture of e2e error retransmission.

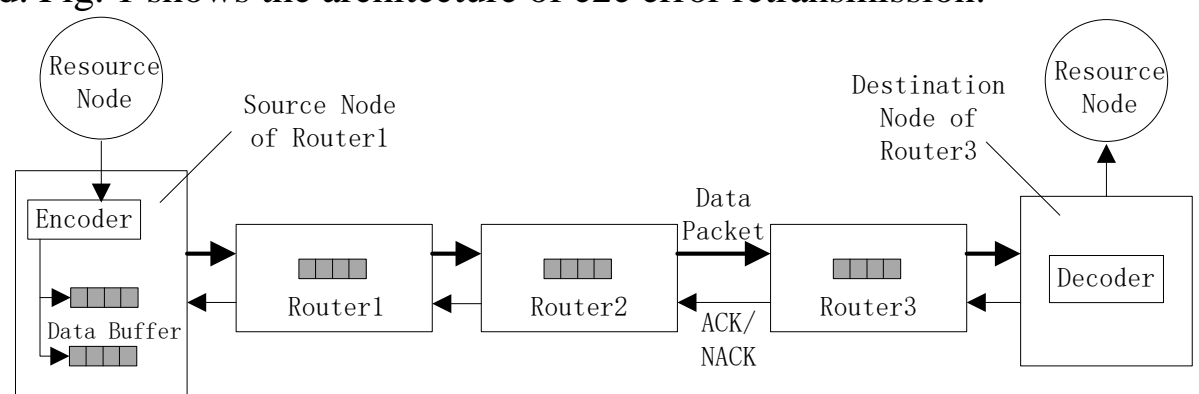

Fig. 1 Architecture of E2E Retransmission Scheme 
Router-to-Router Error Detection and Retransmission. Different from e2e, r2r [10] needs to detect errors at every node the data traverses. R2R scheme has many encoders and decoders.

In this mechanism, data doesn't need to be decoded at the source node, but just be saved into data buffer and sent to the network. Then during the path, it needs to be decoded first and detected errors. If the data is right, it will be encoded and then be transferred to the next router. Otherwise, it will be retransmitted from previous router node and then be detected afresh until the destination gets the right data. Fig. 2 shows the flow of this mechanism.

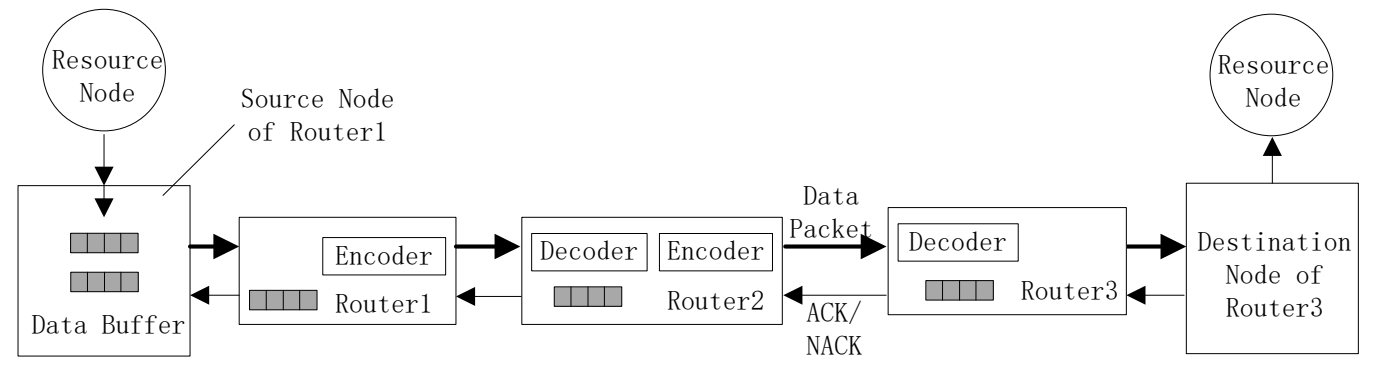

Fig. 2 Architecture of E2E Retransmission Scheme with Many Encoders

Advantages and Disadvantages of the Two Retransmission Schemes. Each of these two error recovery schemes has its advantages and disadvantages [11]. In e2e scheme, the errors of data packet will not be detected until at the destination node, that is to say data packet may have been attainted during the transmission line. So the impact of performance may be high since the data with errors needs to be retransmitted from source node to destination node, which means the delay will be higher. The advantage of e2e is that its energy consumption may be lower because error detection only performed at the destination node.

In r2r scheme, error detection will be performed at every node at the path, so the errors can be found soon and retransmission can be restarted at an earlier time. However, the power and area consumptions will be high because that data needs to be decoded and detected at each node in the path.

All in all, e2e and r2r both have limitations and dominances. But the difference in power and delay between these two schemes gets lower when the error rate increases. So, different retransmission schemes should be taken based on different application scene.

\section{Novel Hybrid Error Detection and Retransmission Mechanism}

Given the practical applications and the analysis of e2e and r2r schemes, taking a hybrid mechanism that utilizes advantages of these two schemes and gives a good tradeoff between cost and reliability becomes very important.

Data Packet Structure and Importance Analysis. In NoC systems, data that transfers in network consists of data packet sent from source node and feedback packet sent from destination node. Data packet is divided into head flit and data flit. Fig. 3 shows the structure of packet.

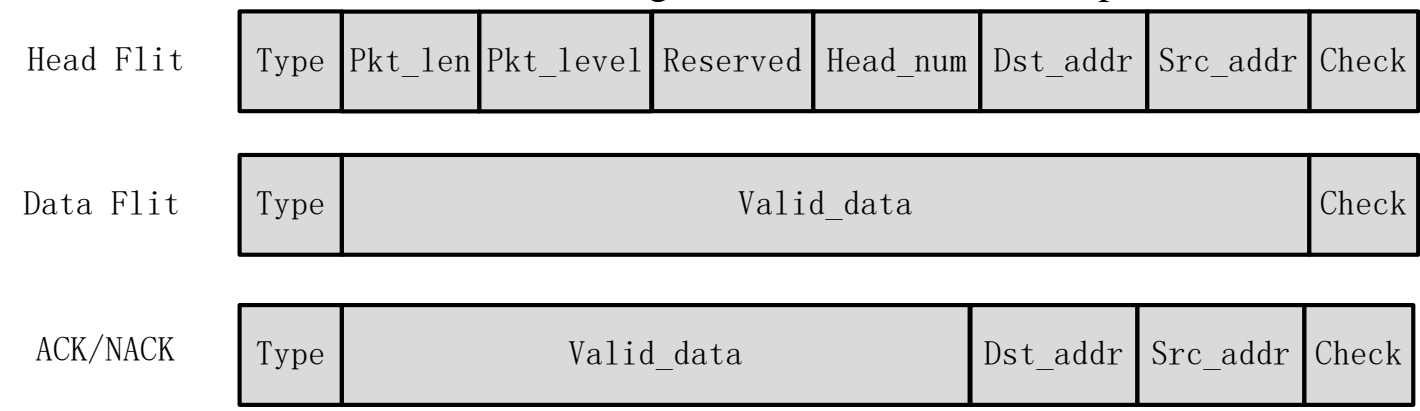

Fig. 3. General Structure of packet and Flit in NoC System

As can be seen in Fig. 3, the head flit contains some important information including destination address, source address, size of data packet and data packet ID. While the data flit just consists of 
valuable data. Compared to data flit, head flit becomes much more significant because it dominates the routing path of this transmission. So, the correctness of head flit must be detected at every node. On the other hand, to the data flit, sometimes the data is not that significant and some little errors can be accepted. In this case, hybrid retransmission scheme can be considered.

Hybrid Error Detection and Retransmission. Hybrid retransmission consists both e2e and r2r schemes. Due to the importance of head flit protection, r2r error detection and retransmission mechanism should be taken. Under this circumstance, head flit will be detected at each node in the network path. If it has errors, then it can be retransmitted in time. This can ensure that the whole data packet transferred to the right destination. Applying this scheme, transfer delay may be reduced when the space environment caused some errors. To the data flits, r2r scheme is abandoned and instead by e2e retransmission. This is because that, e2e just performs error detection at the destination node of the transfer path. It makes the energy consumption lower comparatively.

The Architecture of Novel Hybrid Retransmission Scheme. The structure of this novel hybrid retransmission scheme is shown at fig. 4.To the head flit. First, it will be buffered and then be transferred into the network. In this paper, comparator is used to replace encoder/decoder. To perform the error detection of head flit, three copies of head flit are sent into the comparator module. If two or three of them are the same, then the other one who is different will be modified and the head flit hop to the next node. Otherwise, the node will send a false transfer feedback signal to previous router to get a retransmission of the accurate head flits. This operation will continue until the head flit arrives at the destination node.

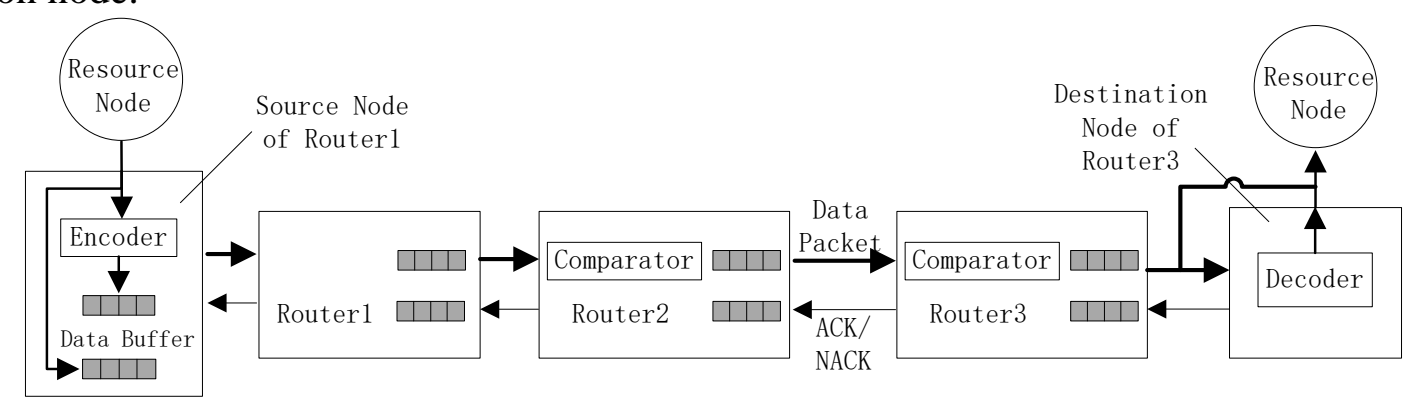

Fig. 4. Architecture of Novel Hybrid Retransmission Scheme

In comparison with head flit, the data flit will be encoded at the source node and saved in buffer separately. Then it will be send to the network. And during the path, the flit just hops from one node to the next. When it arrives at the destination node, it will be decoded and preformed error detection. If the data has errors, it will get a retransmission from the source node until the right one arrive at the destination.

Data Flow of the novel NoC Router. Fig. 5 shows the data flow of the novel router with hybrid retransmission scheme of NoC system. Head of packet and payload of packet will go through different paths to arrive to the destination.

As the data flow presents, the head of the packet will be compared, if the head is right, then all three head data will be buffered at Retrans Reg. Otherwise, a retransmission request will be send to the previous router to get new head data. After the head transferring to the Retrans Reg part, the data will be used to compute the direction that it will be sent to in Rout Compute part. On the other hand, the load of the packet will be buffered into the FIFO part. If the Rout Arbiter permit packet to pass, then all the data will be send to the next router through the Crossbar part.

Function and Simulation Result of the Novel NoC Router. After the novel NoC router is designed, 16 routers are used to constitute a 4*4 2D-Mesh network just like Fig. 6 presents. The router has some tolerant functions to solve the transient faults just as follows. 


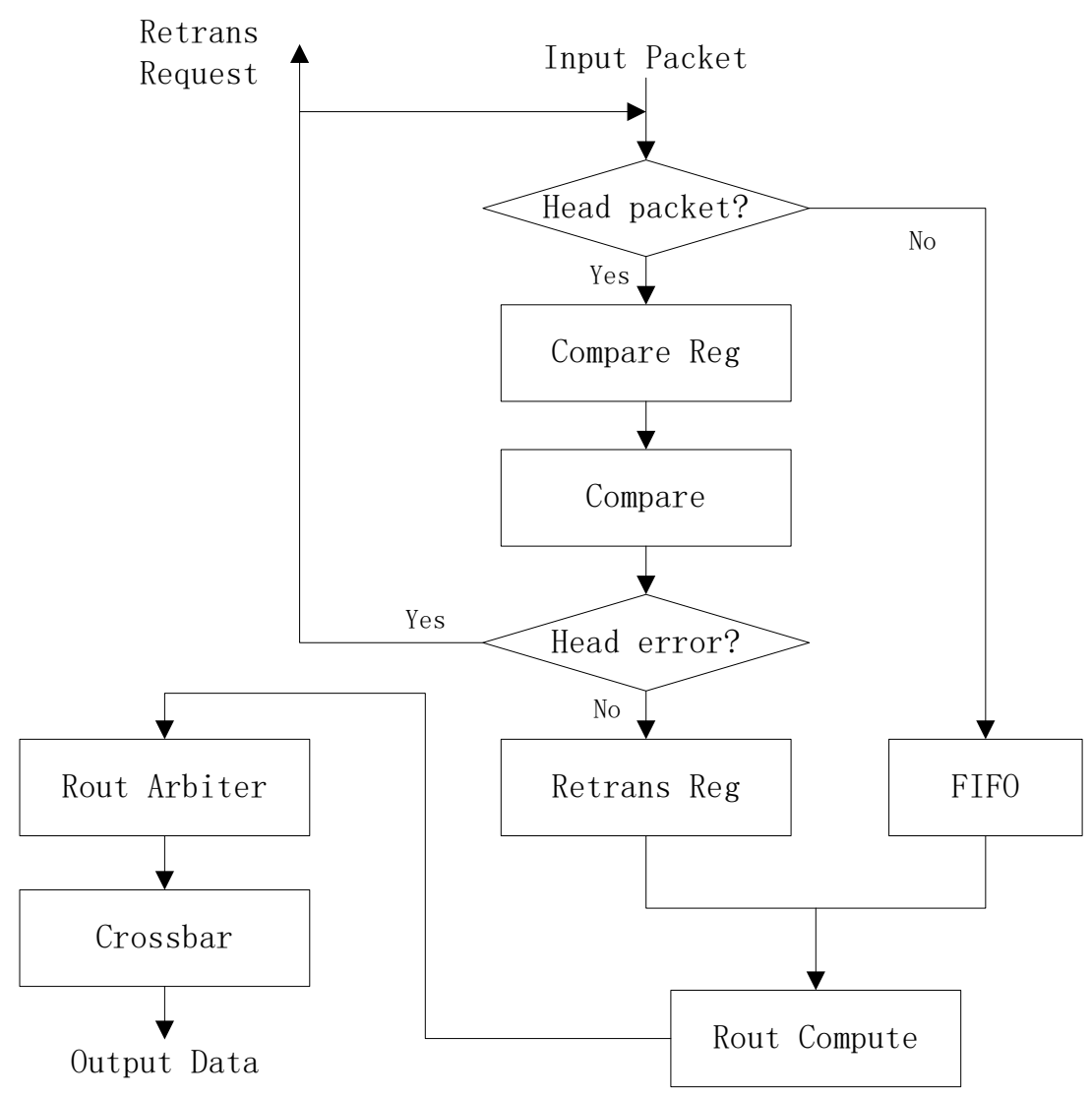

Fig. 5. Data Flow of the Novel NoC Router

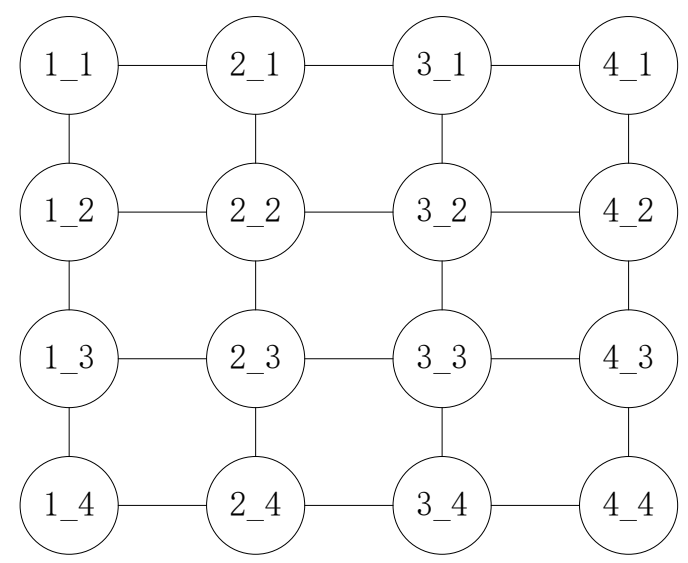

Fig. 6. Structure of the NoC System with Novel Router

- It can detect head flit errors and correct them or get a retransmission of the head because of the triplication redundancy transmission of head flit and r2r retransmission scheme.

- It can solve the conflict of the transmission among the network just for the arbiter scheme of the design.

- Packet infected with transient errors among the transmission path will be retransferred until the end node gets the correct one thanks to the e2d retransmission scheme.

- Packet that lost some flit will also be retransmitted and the authority of the arbiter will be released for other packet to transfer when the deadline is coming just because of the overtime scheme. It not only ensures an unobstructed transmission network, but also assures that destination node can get a correct packet.

Simulation of the design is done to check all the tolerant functions of the novel router, and the result is presented below.

- To simulate the fundamental function of the NoC Router, one packet is sent from router 1_1 to router 2_2 with XY router arithmetic. The result of this transaction is presented at Fig. 7. 


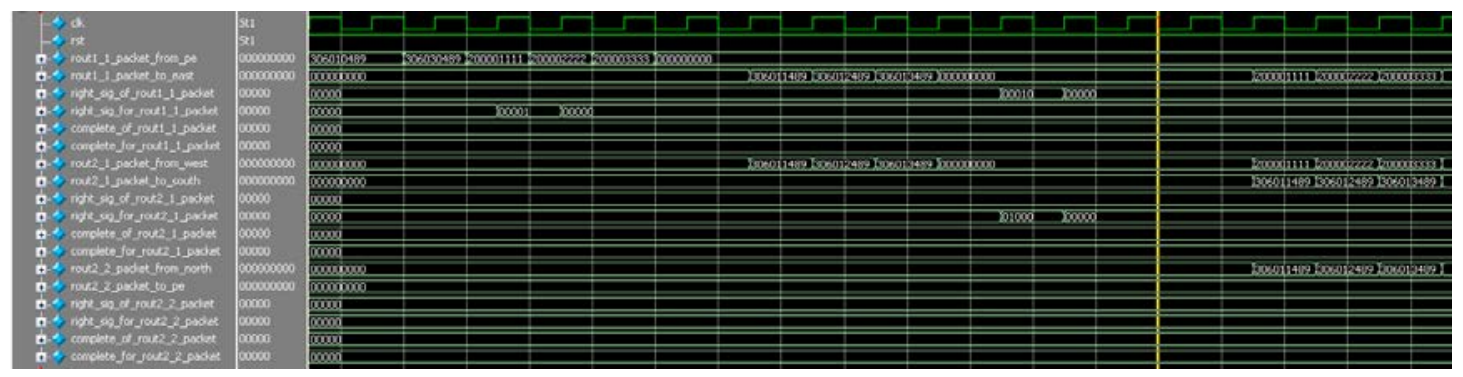

Fig. 7. Simulation1 Result of Novel Router

As can be seen in Figure7, the packet sends from router 1_1 and it is transferred to the destination router 2_2 through router2_1 accurately. To check the fault tolerance scheme, a head data error is imitated at the end node router 2_2, and then the router sends an error feedback signal to previous node router 2_1 and gets a retransmission of the head flit. The process is repeated until router $2 \_2$ gets the right packet.

Fig. 7 shows that packet with some transmission errors can be transferred correctly to the end node just because of the head redundant tolerance scheme. After the transaction, the destination outputs a feedback signal to the source router.

- To simulate the tolerance function of the NoC Router furthermore. One packet is sent from router $1 \_1$ to router 2_2 with XY router arithmetic, while another one is sent from router3_1 to router

$2 \_2$ at the same time. The result of this transaction is presented at Fig. 8.

As Fig. 8 shows, the packet sends from router 1_1 has conflict with the other one sent from router 3_1 at the second node (router 2_1). Owing to the arbiter of the router, both of the two packets arrive at the destination precisely. To check the retransmission scheme, the head of the packet from rout1_1 is supposed to be wrong when it arrives at the destination, then a retransmission request is sent to the previous router to get a new right head.

- To make the tolerance function more comprehensive, the design also considers the short packet issue.

The short packet phenomenon means that the transferred packet is shorter than the length of the packet indicated in the head flit. To check this function, a short packet is send from router 1_1 to router 3_1. As the simulation result presents, the short packet is send to the end node, but for the length shorter, the router 3_1 will wait the remnant all the time. Fortunately, an overtime checking scheme is added in the design. So, the end node router 3_1 sends an overtime feedback to the source node to get a retransmission for an integrated and correct packet.

Feasibility and Predominance of Hybrid Retransmission Scheme. This Hybrid mechanism that consists of both e2e and r2r scheme is acceptant because it can detect the errors of data packet and get a retransmission one. That is to say, the destination node can get the correct packet. On the other hand, it utilizes the advantages of $\mathrm{r} 2 \mathrm{r}$ and e2e, and avoids some limitations of them, which makes a good tradeoff between reliability and cost.

In the hybrid scheme, the comparator is used to make the area and energy consumptions lower.

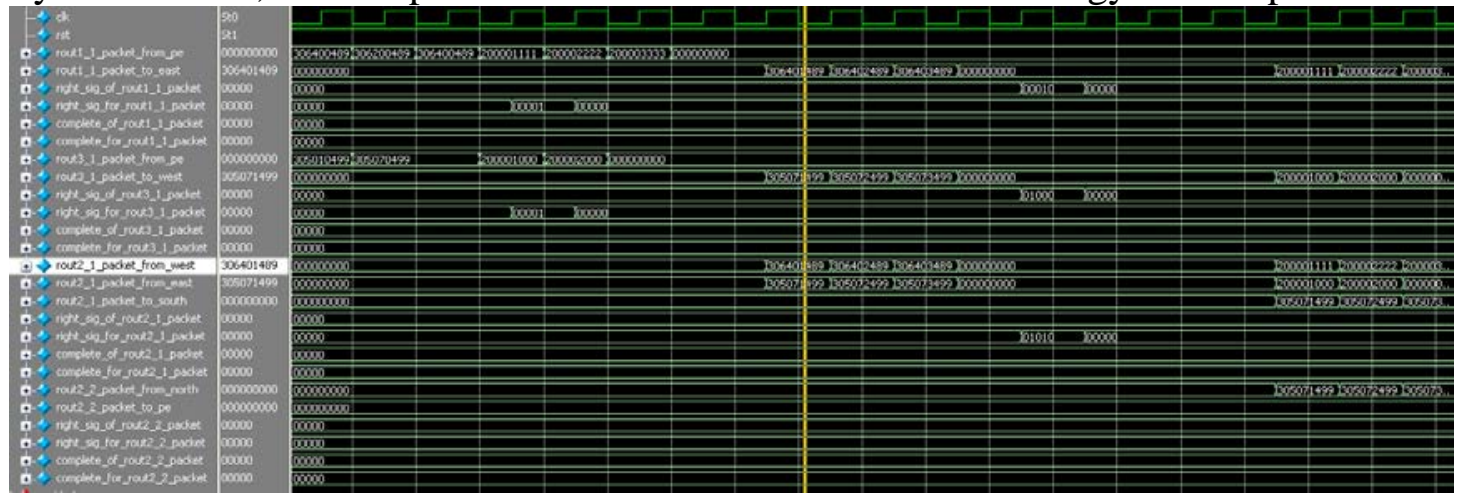

Fig. 8. Simulation2 Result of Novel Router 


\section{Conclusion and Future Work}

The contribution of this paper is the emphasis on the importance of NoC system reliability. Then it reviews some fault-tolerance methods of NoC and summarizes two typical retransmission schemes including r2r and e2e. Especially, the paper presents a novel hybrid retransmission mechanism. It not only can guarantee the integrity of the data packet that transmitted, but also consider the energy and chip area consumptions. However, for some reasons, the final performance of the novel fault-tolerant router has not been finished. The paper just presents its design and some simulation results. More performance analysis work needs to be promoted.

NoC system is now powerful enough to be widely used in MPSoC. The reliability is one of the most important problems that should be taken care. More research should be done about the fault-tolerant routing mechanism of NoC architecture especially the novel hybrid retransmission scheme.

\section{Reference}

[1] A. Hemani, A. Jantseh, S. Kumar, et al, Network on a Chip: Architecture for Billion Transistor Ear. In: Proc of the IEEE NorChip Conference. London Britain: IEEE Press, 2000, 257-263.

[2] L. Benini, G. Micheli, Networks on Chips: A New SoC Paradigm. IEEE Computer, vol. 35, pp. 70-78, Jan. 2002.

[3] P. Vellanki, Quality-of-Service and Error Control Techniques for Network-on-Chip Architectures. In: Great Lakes Symposium on VLSI (GVLSI’04), PP. 45-50, 2004.

[4] H. Zimme, A. Jantsch. A Fault Model Notation and Error-Control Scheme for Switch-to-Switch Buses in a Network-on-Chip, First International Conference on Hardware/Software Codesign and Systems Synthesis, New York, NY, USA, IEEE, ACM, pp. 188-193.

[5] D. Bertozzi, L. Benini, Xpipes: a network-on-chip architecture for gigascale systems-on-chip, Circuits and Systems Magazine, IEEE, vol. 4, no. 2, pp. 18- 31, 2004.

[6] S. Murali, T. Theocharides, N. Vijaykrishnan, et al. Fault Tolerant Algorithms for Network-On-Chip Interconnect. Computer Society Annual Symposium on VLSI. IEEE Comput. Soc, pp. 46-51.

[7] H. Zhu, P. P. Pande, A. Ganguly, et al. Energy Reduction through Crosstalk Avoidance Coding in NoC Paradigm. 9th EUROMICRO Conference on Digital System Design: Architectures, Methods and Tools, pages: 7pp, Los Alamitos, CA, USA, 2006. IEEE Computer, SoC.

[8] J. Hellerstein, Y. Diao, S. Parekh, et al. Feedback Control of Computing Systems, John Wiley \& Sons, 2004.

[9] Z. Hui, K. Mahmut, J. Mary. Exploring Performance-Power Tradeoffs in Providing Reliability for NoC-Based MPSoCs. 12th International Symposium on Quality Electronic Design, ISQED, 2011.

[10] A. H. Lucas, F. G. Moraes. Crosstalk Fault Tolerant NoC - Design and Evaluation. 17th IFIP International Conference on Very Large Scale Integration, VLSI-SoC, pages, 115-120, 2009.

[11] P. Ahmad, G. M. Seyed. Reliability \& Performance Modeling to Speed-Up the NoC Design. 15th IEEE Pacific Rim International Symposium on Dependable Computing, pages, 1-6, 2009. 